PROCEEDINGS OF THE

AMERICAN MATHEMATICAL SOCIETY

Volume 127, Number 3, March 1999, Pages 701-708

S $0002-9939(99) 04647-X$

\title{
MODULAR VARIETIES WITH THE FRASER-HORN PROPERTY
}

\author{
DIEGO VAGGIONE
}

(Communicated by Carl Jockusch)

\begin{abstract}
The notion of central idempotent elements in a ring can be easily generalized to the setting of any variety with the property that proper subalgebras are always nontrivial. We will prove that if such a variety is also congruence modular, then it has factorable congruences, i.e., it has the FraserHorn property. (This property is well known to have major implications for the structure theory of the algebras in the variety.)
\end{abstract}

By a variety with $\overrightarrow{0}$ and $\overrightarrow{1}$ we understand a variety $\mathcal{V}$ for which there exist unary terms $0_{1}(w), \ldots, 0_{n}(w), 1_{1}(w), \ldots, 1_{n}(w)$ such that

$$
\mathcal{V} \models \overrightarrow{0}(w)=\overrightarrow{1}(w) \rightarrow x=y,
$$

where $\overrightarrow{0}=\left(0_{1}, \ldots, 0_{n}\right)$ and $\overrightarrow{1}=\left(1_{1}, \ldots, 1_{n}\right){ }^{1}$

Indeed this condition is equivalent to the more familiar property that no nontrivial algebra of $\mathcal{V}$ has a trivial subalgebra (combine [17, Lemma 3] with [12]).

If $\lambda \in A \in \mathcal{V}$, then we say that $\vec{e} \in A^{n}$ is a $\lambda$-central element of $A$ if there exists an isomorphism $A \rightarrow A_{1} \times A_{2}$ such that

$$
\begin{aligned}
& \lambda \rightarrow\left(\lambda_{1}, \lambda_{2}\right), \\
& \vec{e} \rightarrow\left(\left(0_{1}\left(\lambda_{1}\right), 1_{1}\left(\lambda_{2}\right)\right), \ldots,\left(0_{n}\left(\lambda_{1}\right), 1_{n}\left(\lambda_{2}\right)\right)\right) .
\end{aligned}
$$

It is well known that the central elements of a ring with identity are just the central idempotent ones. To give another example, an element in a bounded lattice is central iff it is neutral and complemented [9].

A variety $\mathcal{V}$ has the Fraser-Horn property if each $\theta \in \operatorname{Con}\left(A_{1} \times A_{2}\right), A_{1}, A_{2} \in \mathcal{V}$, is of the form $\theta_{1} \times \theta_{2}$, with $\theta_{i} \in \operatorname{Con}\left(A_{i}\right), i=1,2$. This property is well known to have major implications for the structure theory of the algebras in the variety (see for example, [1], [2], [3], [7], [8], [14]).

In [15] and [16] we used central elements for characterizing the varieties with the Fraser-Horn property for which the Pierce sheaf [13] (is Hausdorff and) has only directly indecomposable stalks. In this paper we will use central elements to prove the following

Theorem 1. If $\mathcal{V}$ is a congruence modular variety such that no non-trivial algebra of $\mathcal{V}$ has a trivial subalgebra, then $\mathcal{V}$ has the Fraser-Horn property.

Also, we prove a result which shows that in congruence modular varieties with $\overrightarrow{0}$ and $\overrightarrow{1}$ the central elements have the fundamental properties of central elements

Received by the editors April 24, 1997 and, in revised form, July 7, 1997.

1991 Mathematics Subject Classification. Primary 08A05, 08B10.

This research was supported by CONICOR and SECYT (UNC).

${ }^{1}$ If the language of $\mathcal{V}$ has a constant, then we can remove the variable $w$.

(C)1999 American Mathematical Society 
in rings with identity or bounded lattices (Proposition 4). We conclude the paper giving an example to show that even for the congruence distributive case, the property " $\vec{e}$ is $\lambda$-central" is not definable by a set of formulas which are either universal or existential.

For background on universal algebra and model theory we refer the reader to [4], [11] and [5]. Let $A$ be an algebra. If $\vec{a}, \vec{b} \in A^{n}$, then we use $\theta^{A}(\vec{a}, \vec{b})$ to denote the congruence generated by the set $\left\{\left(a_{k}, b_{k}\right): 1 \leq k \leq n\right\}$. $\nabla^{A}$ is the universal congruence on $A$ and $\Delta^{A}$ is the trivial congruence on $A$. Con $(A)$ denotes the congruence lattice of $A$. A pair of congruences $\theta, \delta \in \operatorname{Con}(A)$, which satisfies $\theta \cap \delta=\Delta^{A}$ and $\theta \circ \delta=\nabla^{A}$ is called a pair of complementary factor congruences.

For an algebra $A$ in a variety with $\overrightarrow{0}$ and $\overrightarrow{1}$, we will use $Z_{\lambda}(A)$ to denote the set of $\lambda$-central elements of $A$. If $\vec{a} \in A^{n}$ and $\vec{b} \in B^{n}$, then we will use $[\vec{a}, \vec{b}]$ to denote the $n$-tuple $\left(\left(a_{1}, b_{1}\right), \ldots,\left(a_{n}, b_{n}\right)\right)$.

Lemma 2. Let $\mathcal{V}$ be a congruence modular variety with $\overrightarrow{0}$ and $\overrightarrow{1}$. Let $\lambda \in A \in \mathcal{V}$. Let $P=\{(\theta, \delta): \theta, \delta$ is a pair of complementary factor congruences of $A\}$.

(a) The map

$$
\begin{aligned}
Z_{\lambda}(A) & \rightarrow P \\
\vec{e} & \rightarrow\left(\theta^{A}(\overrightarrow{0}(\lambda), \vec{e}), \theta^{A}(\overrightarrow{1}(\lambda), \vec{e})\right)
\end{aligned}
$$

is bijective.

$$
\text { (b) } \vec{e} \in Z_{\lambda}(A) \text { iff }\left(\theta^{A}(\overrightarrow{0}(\lambda), \vec{e}), \theta^{A}(\overrightarrow{1}(\lambda), \vec{e})\right) \in P \text {. }
$$

Proof. We will see that the map of (a) is well defined. Let $\vec{e} \in Z_{\lambda}(A)$. We can suppose that $A=A_{1} \times A_{2}, \lambda=\left(\lambda_{1}, \lambda_{2}\right)$ and $\vec{e}=\left[\overrightarrow{0}\left(\lambda_{1}\right), \overrightarrow{1}\left(\lambda_{2}\right)\right]$. Let $\pi_{1}$ and $\pi_{2}$ be the kernels of the canonical projections. Note that $\theta^{A}(\overrightarrow{0}(\lambda), \vec{e}) \subseteq \pi_{1}$ and $\theta^{A}(\overrightarrow{1}(\lambda), \vec{e}) \subseteq$ $\pi_{2}$. Furthermore note that $\theta^{A}(\overrightarrow{0}(\lambda), \vec{e}) \vee \theta^{A}(\overrightarrow{1}(\lambda), \vec{e}) \supseteq \theta^{A}(\overrightarrow{0}(\lambda), \overrightarrow{1}(\lambda))=\nabla^{A}$. Thus by modularity we have that $\theta^{A}(\overrightarrow{0}(\lambda), \vec{e})=\pi_{1}$ and $\theta^{A}(\overrightarrow{1}(\lambda), \vec{e})=\pi_{2}$. Next, we will prove that the map of (a) is onto. Let $(\theta, \delta) \in P$. Let $\vec{e}$ be the element of $A^{n}$ such that $\left(0_{i}(\lambda), e_{i}\right) \in \theta$ and $\left(e_{i}, 1_{i}(\lambda)\right) \in \delta$, for every $i=1, \ldots, n$. Since $\theta^{A}(\overrightarrow{0}(\lambda), \vec{e}) \subseteq \theta$ and $\theta^{A}(\overrightarrow{1}(\lambda), \vec{e}) \subseteq \delta$, the same argument as above produces $\theta^{A}(\overrightarrow{0}(\lambda), \vec{e})=\theta$ and $\theta^{A}(\overrightarrow{1}(\lambda), \vec{e})=\delta$ and hence the map is onto. The injectivity and (b) are easy to check.

Let $\mathcal{V}$ be a variety. For a set $X$ of variables we use $F(X)$ to denote the free algebra of $\mathcal{V}$ freely generated by $X$.

Lemma 3. Let $\mathcal{V}$ be a congruence modular variety with $\overrightarrow{0}$ and $\overrightarrow{1}$.

(a) There is a set $\Sigma$ of formulas of the form $\forall \exists \wedge p=q$ such that for every $\vec{e} \in A^{n}, \lambda \in A \in \mathcal{V}$ we have

$\vec{e}$ is $\lambda$-central iff $A \models \varphi(\vec{e}, \lambda)$, for every $\varphi \in \Sigma$.

(b) There is a formula $\varphi$ of the form $\bigwedge p=q$ such that for every $A=A_{1} \times A_{2}$ and $\lambda=\left(\lambda_{1}, \lambda_{2}\right) \in A$, we have

$(x, y) \in \operatorname{ker}\left(\pi_{1}\right)$ iff $A \models \varphi(\vec{e}, x, y, \lambda)$, with $\vec{e}=\left(\left(0_{1}\left(\lambda_{1}\right), 1_{1}\left(\lambda_{2}\right)\right), \ldots,\left(0_{n}\left(\lambda_{1}\right), 1_{n}\left(\lambda_{2}\right)\right)\right)$ and $\pi_{1}: A \rightarrow A_{1}$ the canonical projection.

Proof. Let $X=\left\{x, y, z_{1}, \ldots, z_{n}, w\right\}$. Let $\theta=\theta^{F(X)}(\overrightarrow{1}(w), \vec{z}), \delta=\theta^{F(X)}(\overrightarrow{0}(w), \vec{z})$ and $\gamma=\theta^{F(X)}(x, y)$. Since $\mathcal{V}$ is congruence modular, we have 


$$
\begin{aligned}
(x, y) & \in(\theta \vee \gamma) \cap(\delta \vee \gamma) \\
& =(\theta \vee \gamma) \cap((\delta \vee \theta) \cap(\delta \vee \gamma)) \\
& =(\theta \vee \gamma) \cap(\delta \vee(\theta \cap(\delta \vee \gamma))) \\
& =((\theta \vee \gamma) \cap \delta) \vee(\theta \cap(\delta \vee \gamma)) \\
& =(\delta \cap(\theta \vee \gamma)) \vee(\theta \cap(\delta \vee \gamma)),
\end{aligned}
$$

which implies that there exist $(n+3)$-ary terms $v_{1}, \ldots, v_{k}$, such that:

$v_{1}(\vec{z}, x, y, w)=x$,

$v_{k}(\vec{z}, x, y, w)=y$,

$\left(v_{i}(\vec{z}, x, y, w), v_{i+1}(\vec{z}, x, y, w)\right) \in(\delta \cap(\theta \vee \gamma))$, for $1 \leq i \leq k-1, i$ odd,

$\left(v_{i}(\vec{z}, x, y, w), v_{i+1}(\vec{z}, x, y, w)\right) \in(\theta \cap(\delta \vee \gamma))$, for $1 \leq i \leq k-1$, $i$ even.

Thus we have that the following are identities of $\mathcal{V}$ :

(I1) $v_{1}(\vec{z}, x, y, w)=x$.

(I2) $v_{k}(\vec{z}, x, y, w)=y$.

(I3) $v_{i}(\overrightarrow{0}(w), x, y, w)=v_{i+1}(\overrightarrow{0}(w), x, y, w)$,

$$
v_{i}(\overrightarrow{1}(w), x, x, w)=v_{i+1}(\overrightarrow{1}(w), x, x, w),
$$

for $1 \leq i \leq k-1, i$ odd.

(I4) $v_{i}(\overrightarrow{1}(w), x, y, w)=v_{i+1}(\overrightarrow{1}(w), x, y, w)$,

$$
v_{i}(\overrightarrow{0}(w), x, x, w)=v_{i+1}(\overrightarrow{0}(w), x, x, w),
$$

for $1 \leq i \leq k-1, i$ even.

Next, let

$$
\begin{aligned}
& \Lambda(\vec{z}, x, y, w)=\bigwedge_{1 \leq i \leq k-1, i \text { even }} v_{i}(\vec{z}, x, y, w)=v_{i+1}(\vec{z}, x, y, w), \\
& R(\vec{z}, x, y, w)=\bigwedge_{1 \leq i \leq k-1, i \text { odd }} v_{i}(\vec{z}, x, y, w)=v_{i+1}(\vec{z}, x, y, w) .
\end{aligned}
$$

We will prove

(1) For every $\vec{e} \in A^{n}, \lambda \in A \in \mathcal{V}$, if $\theta^{A}(\overrightarrow{0}(\lambda), \vec{e}) \cap \theta^{A}(\overrightarrow{1}(\lambda), \vec{e})=\Delta^{A}$, then $\theta^{A}(\overrightarrow{0}(\lambda), \vec{e})=\left\{(a, b) \in A^{2}: A \models \Lambda(\vec{e}, a, b, \lambda)\right\}$, $\theta^{A}(\overrightarrow{1}(\lambda), \vec{e})=\left\{(a, b) \in A^{2}: A=R(\vec{e}, a, b, \lambda)\right\}$.

Using (I1), (I2) and (I3) it can be proved that $A \models \Lambda(\vec{e}, a, b, \lambda)$ implies $(a, b) \in$ $\theta^{A}(\overrightarrow{0}(\lambda), \vec{e})$. If $(a, b) \in \theta^{A}(\overrightarrow{0}(\lambda), \vec{e})$, then, by (I3) and (I4), the equations of $\Lambda(\vec{e}, a, b, \lambda)$ hold modulo $\theta^{A}(\overrightarrow{0}(\lambda), \vec{e}) \cap \theta^{A}(\overrightarrow{1}(\lambda), \vec{e})$, which concludes the first equality. The other one is similar.

We observe that (b) follows from (1). Next, let $X=\left\{z_{1}, \ldots, z_{n}, x, y, z, w\right\}, F=$ $F(X)$ and let $\gamma$ be the following congruence:

$$
\bigvee_{\substack{1 \leq i \leq k-1, i \text { even }}} \theta^{F}\left(v_{i}(\vec{z}, x, y, w), v_{i+1}(\vec{z}, x, y, w)\right) \vee \theta^{F}\left(v_{i}(\vec{z}, y, z, w), v_{i+1}(\vec{z}, y, z, w)\right)
$$

Note that, by (I4), $\gamma \subseteq \theta^{F}(\overrightarrow{1}(w), \vec{z})$. Let $1 \leq i \leq k-1, i$ even. By (I3) we have

$\left(v_{j}(\vec{z}, x, y, w), v_{j+1}(\vec{z}, x, y, w)\right) \in \theta^{F}(\overrightarrow{0}(w), \vec{z}), j$ odd, and then

$\left(v_{i}\left(\vec{z}, v_{1}(\vec{z}, x, y, w), z, w\right), v_{i}\left(\vec{z}, v_{k}(\vec{z}, x, y, w), z, w\right)\right) \in \gamma \vee \theta^{F}(\overrightarrow{0}(w), \vec{z})$,

$\left(v_{i+1}\left(\vec{z}, v_{k}(\vec{z}, x, y, w), z, w\right), v_{i+1}\left(\vec{z}, v_{1}(\vec{z}, x, y, w), z, w\right)\right) \in \gamma \vee \theta^{F}(\overrightarrow{0}(w), \vec{z})$.

Since

$\left(v_{i}\left(\vec{z}, v_{k}(\vec{z}, x, y, w), z, w\right), v_{i+1}\left(\vec{z}, v_{k}(\vec{z}, x, y, w), z, w\right)\right) \in \gamma$

we have that

$$
\begin{aligned}
& \left(v_{i}(\vec{z}, x, z, w), v_{i+1}(\vec{z}, x, z, w)\right) \\
& \quad=\left(v_{i}\left(\vec{z}, v_{1}(\vec{z}, x, y, w), z, w\right), v_{i+1}\left(\vec{z}, v_{1}(\vec{z}, x, y, w), z, w\right)\right) \\
& \quad \in \gamma \vee \theta^{F}(\overrightarrow{0}(w), \vec{z})
\end{aligned}
$$


By (I4)

$$
\left(v_{i}(\vec{z}, x, z, w), v_{i+1}(\vec{z}, x, z, w)\right) \in \theta^{F}(\overrightarrow{1}(w), \vec{z})
$$

and hence, by modularity we have

$$
\left(v_{i}(\vec{z}, x, z, w), v_{i+1}(\vec{z}, x, z, w)\right) \in \gamma \vee\left(\theta^{F}(\overrightarrow{0}(w), \vec{z}) \cap \theta^{F}(\overrightarrow{1}(w), \vec{z})\right) .
$$

Thus there exist terms $P_{i, l}^{\text {tra }}(\vec{z}, x, y, z, w), 1 \leq l \leq h_{i}$, such that the following hold in $\mathcal{V}$ :

$$
\begin{aligned}
& P_{i, 1}^{\text {tra }}(\vec{z}, x, y, z, w)=v_{i}(\vec{z}, x, z, w), \\
& P_{i, h_{i}}^{\text {tra }}(\vec{z}, x, y, z, w)=v_{i+1}(\vec{z}, x, z, w), \\
& \Lambda(\vec{z}, x, y, w) \wedge \Lambda(\vec{z}, y, z, w) \rightarrow \bigwedge_{1 \leq l \leq h_{i}-1,} P_{i, l}^{\text {tra }}(\vec{z}, x, y, z, w)=P_{i, l+1}^{\text {tra }}(\vec{z}, x, y, z, w), \\
& \bigwedge_{1 \leq l \leq h_{i}-1, l \text { even }} P_{i, l}^{\text {tra }}(\overrightarrow{0}(w), x, y, z, w)=P_{i, l+1}^{\text {tra }}(\overrightarrow{0}(w), x, y, z, w), \\
& \bigwedge_{1 \leq l \leq h_{i}-1, l \text { even }}^{\text {tra }} P_{i, l}^{\text {tra }}(\overrightarrow{1}(w), x, y, z, w)=P_{i, l+1}^{\text {tra }}(\overrightarrow{1}(w), x, y, z, w) .
\end{aligned}
$$

Let

$$
\operatorname{TRANS} S_{\Lambda}(\vec{z}, w)=\forall x, y, z \bigwedge_{\substack{1 \leq i \leq k-1, i \text { even } \\ 1 \leq l \leq h_{i}-1, l \text { even }}} P_{i, l}^{\operatorname{tra}}(\vec{z}, x, y, z, w)=P_{i, l+1}^{\operatorname{tra}}(\vec{z}, x, y, z, w) .
$$

We observe that for every $\vec{e} \in A^{n}, \lambda \in A \in \mathcal{V}$ :

(2) If $\theta^{A}(\overrightarrow{0}(\lambda), \vec{e}) \cap \theta^{A}(\overrightarrow{1}(\lambda), \vec{e})=\Delta^{A}$, then $A=T R A N S_{\Lambda}(\vec{e}, \lambda)$.

(3) $A=T R A N S_{\Lambda}(\vec{e}, \lambda) \rightarrow(\forall x, y, z \Lambda(\vec{e}, x, y, \lambda) \wedge \Lambda(\vec{e}, y, z, \lambda) \rightarrow \Lambda(\vec{e}, x, z, \lambda))$.

Using a similar argument we can define a formula $S Y M_{\Lambda}$ such that

(4) If $\theta^{A}(\overrightarrow{0}(\lambda), \vec{e}) \cap \theta^{A}(\overrightarrow{1}(\lambda), \vec{e})=\Delta^{A}$, then $A=S Y M_{\Lambda}(\vec{e}, \lambda)$.

(5) $A=S Y M_{\Lambda}(\vec{e}, \lambda) \rightarrow(\forall x, y \Lambda(\vec{e}, x, y, \lambda) \rightarrow \Lambda(\vec{e}, y, x, \lambda))$.

Next, let $f$ be a $m$-ary function symbol. Let $X=\left\{z_{1}, \ldots, z_{n}, x_{1}, \ldots, x_{m}, y_{1}, \ldots, y_{m}, w\right\}$ and $F=F(X)$. Let

$$
\gamma=\bigvee_{\substack{1 \leq i \leq k-1, i \text { even } \\ 1 \leq j \leq m}} \theta^{F}\left(v_{i}\left(\vec{z}, x_{j}, y_{j}, w\right), v_{i+1}\left(\vec{z}, x_{j}, y_{j}, w\right)\right)
$$

Let $1 \leq i \leq k-1, i$ even. By (I3) we have

$$
\left(v_{l}\left(\vec{z}, x_{j}, y_{j}, w\right), v_{l+1}\left(\vec{z}, x_{j}, y_{j}, w\right)\right) \in \theta^{F}(\overrightarrow{0}(w), \vec{z}), l \text { odd }, 1 \leq j \leq m,
$$
and then

$$
\begin{aligned}
& \left(v_{i}(\vec{z}, f(\vec{x}), f(\vec{y}), w), v_{i}(\vec{z}, f(\vec{y}), f(\vec{y}), w)\right) \in \gamma \vee \theta^{F}(\overrightarrow{0}(w), \vec{z}), \\
& \left(v_{i+1}(\vec{z}, f(\vec{y}), f(\vec{y}), w), v_{i+1}(\vec{z}, f(\vec{x}), f(\vec{y}), w)\right) \in \gamma \vee \theta^{F}(\overrightarrow{0}(w), \vec{z}) .
\end{aligned}
$$

$\left(v_{i}(\vec{z}, f(\vec{y}), f(\vec{y}), w), v_{i+1}(\vec{z}, f(\vec{y}), f(\vec{y}), w)\right) \in \theta^{F}(\overrightarrow{0}(w), \vec{z})$, which implies that

$$
\begin{aligned}
\left(v_{i}(\vec{z}, f(\vec{x}), f(\vec{y}), w), v_{i+1}(\vec{z}, f(\vec{x}), f(\vec{y}), w)\right) & \in\left(\gamma \vee \theta^{F}(\overrightarrow{0}(w), \vec{z})\right) \cap \theta^{F}(\overrightarrow{1}(w), \vec{z}) \\
& =\gamma \vee\left(\theta^{F}(\overrightarrow{0}(w), \vec{z}) \cap \theta^{F}(\overrightarrow{1}(w), \vec{z})\right) .
\end{aligned}
$$

Thus there exist terms $P_{i, l}^{f}(\vec{z}, \vec{x}, \vec{y}, w), 1 \leq l \leq o_{i}$, such that the following hold in $\mathcal{V}$ :

$$
\begin{aligned}
& P_{i, 1}^{f}(\vec{z}, \vec{x}, \vec{y}, w)=v_{i}(\vec{z}, f(\vec{x}), f(\vec{y}), w), \\
& P_{i, o_{i}}^{f}(\vec{z}, \vec{x}, \vec{y}, w)=v_{i+1}(\vec{z}, f(\vec{x}), f(\vec{y}), w), \\
& \bigwedge_{i=1}^{i=m} \Lambda\left(\vec{z}, x_{i}, y_{i}, w\right) \rightarrow \bigwedge_{1 \leq l \leq o_{i}-1, l \text { odd }}^{f} P_{i, l}^{f}(\vec{z}, \vec{x}, \vec{y}, w)=P_{i, l+1}^{f}(\vec{z}, \vec{x}, \vec{y}, w), \\
& \bigwedge_{1 \leq l \leq o_{i}-1, l \text { even }}^{\wedge} P_{i, l}^{f}(\overrightarrow{0}(w), \vec{x}, \vec{y}, w)=P_{i, l+1}^{f}(\overrightarrow{0}(w), \vec{x}, \vec{y}, w), \\
& \bigwedge_{1 \leq l \leq o_{i}-1, l \text { even }} P_{i, l}^{f}(\overrightarrow{1}(w), \vec{x}, \vec{y}, w)=P_{i, l+1}^{f}(\overrightarrow{1}(w), \vec{x}, \vec{y}, w) .
\end{aligned}
$$


Let

$$
f-P R E S_{\Lambda}(\vec{z}, w)=\forall \vec{x}, \vec{y} \bigwedge_{\substack{1 \leq i \leq k-1, i \text { even } \\ 1 \leq l \leq o_{i}-1, l \text { even }}} P_{i, l}^{f}(\vec{z}, \vec{x}, \vec{y}, w)=P_{i, l+1}^{f}(\vec{z}, \vec{x}, \vec{y}, w) .
$$

We note that for every $\vec{e} \in \bar{A}^{n}, A \in \mathcal{V}$ :

(6) If $\theta(\overrightarrow{0}(w), \vec{e}) \cap \theta(\vec{e}, \overrightarrow{1}(\lambda))=\Delta^{A}$, then $A=f-P R E S_{\Lambda}(\vec{e}, \lambda)$.

(7) $A=f-P R E S_{\Lambda}(\vec{e}, \lambda) \rightarrow\left(\forall \vec{x}, \vec{y} \bigwedge_{i=1}^{i=m} \Lambda\left(\vec{e}, x_{i}, y_{i}, \lambda\right) \rightarrow \Lambda(\vec{e}, f(\vec{x}), f(\vec{y}), \lambda)\right)$.

In a similar manner, formulas $T R A N S_{R}, S Y M_{R}$ and $f-P R E S_{R}$ can be defined with the corresponding properties with respect to $R$. Let

$$
\begin{aligned}
& \Sigma_{1}=\left\{\forall x \Lambda(\vec{z}, x, x, w), S Y M_{\Lambda}(\vec{z}, w), T R A N S_{\Lambda}(\vec{z}, w)\right\}, \\
& \Sigma_{2}=\left\{\forall x R(\vec{z}, x, x, w), S Y M_{R}(\vec{z}, w), \operatorname{TRANS} S_{R}(\vec{z}, w)\right\}, \\
& \Sigma_{3}=\left\{f-P R E S_{\Lambda}(\vec{z}, w), f-P R E S_{R}(\vec{z}, w): f \text { is a function symbol }\right\}, \\
& \Sigma_{4}=\left\{\bigwedge_{i=1}^{i=n} \Lambda\left(\vec{z}, 0_{i}(w), z_{i}, w\right), \bigwedge_{i=1}^{i=n} R\left(\vec{z}, 1_{i}(w), z_{i}, w\right)\right\}, \\
& \Sigma_{5}=\{\forall x, y \exists z \Lambda(\vec{z}, x, z, w) \wedge R(\vec{z}, z, y, w)\} .
\end{aligned}
$$

We will prove that $\Sigma=\Sigma_{1} \cup \Sigma_{2} \cup \Sigma_{3} \cup \Sigma_{4} \cup \Sigma_{5}$ is the required set of axioms. If $\vec{e} \in Z_{\lambda}(A)$, then by (1),(2),(4) and (6) we have that $A \models \varphi(\vec{e}, \lambda)$, for every $\varphi \in \Sigma$. Suppose that $A=\varphi(\vec{e}, \lambda)$, for every $\varphi \in \Sigma$. Let

$$
\begin{aligned}
& L=\left\{(a, b) \in A^{2}: A \models \Lambda(\vec{e}, a, b, \lambda)\right\}, \\
& D=\left\{(a, b) \in A^{2}: A=R(\vec{e}, a, b, \lambda)\right\} .
\end{aligned}
$$

By (3),(5) and (7), the axioms of $\Sigma_{1} \cup \Sigma_{2} \cup \Sigma_{3}$ guarantee that $L$ and $D$ are congruences of $A$. By (I1) and (I2), we have $\mathcal{V} \models \Lambda(\vec{z}, x, y, w) \wedge R(\vec{z}, x, y, w) \rightarrow x=y$ and hence $L \cap D=\Delta^{A}$. The axioms of $\Sigma_{5}$ imply that $L \circ D=\nabla^{A}$ and the axioms of $\Sigma_{4}$ guarantee that $\theta^{A}(\overrightarrow{0}(\lambda), \vec{e}) \subseteq L$ and $\theta^{A}(\overrightarrow{1}(\lambda), \vec{e}) \subseteq D$. By modularity we have that $\theta^{A}(\overrightarrow{0}(\lambda), \vec{e})=L$ and $\theta^{A}(\overrightarrow{1}(\lambda), \vec{e})=D$, and hence Lemma 2 implies that $\vec{e} \in Z_{\lambda}(A)$.

Remark. For the case in which we have an equational proof of $\{$ identities of $\mathcal{V}\} \cup$ $\{\overrightarrow{0}(w)=\overrightarrow{1}(w)\} \vdash x=y$, we can use the techniques from [10] to construct (via the Day-terms [6]) the axioms of $\Sigma .^{2}$

Proof of Theorem 1. Since no non-trivial algebra of $\mathcal{V}$ has a trivial subalgebra, combining [17, Lemma 3] with [12] we obtain that $\mathcal{V}$ is a variety with $\overrightarrow{0}$ and $\overrightarrow{1}$. Let $\theta, \delta$ be a pair of complementary factor congruences of an algebra $A \in \mathcal{V}$. By Lemma 2 , there exists $\vec{e} \in Z_{\lambda}(A)$, such that $\theta=\theta^{A}(\overrightarrow{0}(\lambda), \vec{e})$ and $\delta=\theta^{A}(\overrightarrow{1}(\lambda), \vec{e})$. Let $\sigma \in$ $\operatorname{Con}(A)$. Note that for every $\vec{a}, \vec{b} \in A^{n}$, we have $\theta^{A / \sigma}(\vec{a} / \sigma, \vec{b} / \sigma)=\left(\theta^{A}(\vec{a}, \vec{b}) \vee \sigma\right) / \sigma$, where $\vec{a} / \sigma=\left(a_{1} / \sigma, \ldots, a_{n} / \sigma\right)$. By (a) of Lemma $3, \vec{e} / \sigma \in Z_{\lambda / \sigma}(A / \sigma)$, and therefore

$$
\begin{aligned}
\Delta^{A / \sigma}= & \theta^{A / \sigma}(\overrightarrow{0}(\lambda / \sigma), \vec{e} / \sigma) \cap \theta^{A / \sigma}(\overrightarrow{1}(\lambda / \sigma), \vec{e} / \sigma) \\
& \left(\theta^{A}(\overrightarrow{0}(\lambda), \vec{e}) \vee \sigma\right) / \sigma \cap\left(\theta^{A}(\overrightarrow{1}(\lambda), \vec{e}) \vee \sigma\right) / \sigma .
\end{aligned}
$$

Thus $(\theta \vee \sigma) \cap(\delta \vee \sigma) \subseteq \sigma$, and hence by [8, Theorem 2] the theorem follows.

For a congruence modular variety with $\overrightarrow{0}$ and $\overrightarrow{1}$, define

$$
\begin{aligned}
& \Lambda(\vec{z}, \vec{x}, \vec{y}, w)=\bigwedge_{i=1}^{i=n} \Lambda\left(\vec{z}, x_{i}, y_{i}, w\right), \\
& R(\vec{z}, \vec{x}, \vec{y}, w)=\bigwedge_{i=1}^{i=n} R\left(\vec{z}, x_{i}, y_{i}, w\right),
\end{aligned}
$$

where $\Lambda(\vec{z}, x, y, w)$ and $R(\vec{z}, x, y, w)$ are as in the proof of Lemma 3 . Note that by (1) in that proof, for $\vec{e} \in Z_{\lambda}(A)$ we have

$$
\begin{aligned}
& A \models \Lambda(\vec{e}, \vec{a}, \vec{b}, \lambda) \text { iff }\left(a_{i}, b_{i}\right) \in \theta^{A}(\overrightarrow{0}(\lambda), \vec{e}), \text { for every } i=1, \ldots, n, \\
& A \models R(\vec{e}, \vec{a}, \vec{b}, \lambda) \text { iff }\left(a_{i}, b_{i}\right) \in \theta^{A}(\overrightarrow{1}(\lambda), \vec{e}) \text {, for every } i=1, \ldots, n .
\end{aligned}
$$

\footnotetext{
${ }^{2}$ Note that the $v_{i}$ 's of the proof of Lemma 3 provide such an equational proof.
} 
Proposition 4. Let $\mathcal{V}$ be a congruence modular variety with $\overrightarrow{0}$ and $\overrightarrow{1}$.

(a) $\left\langle Z_{\lambda}(A), \vee, \wedge,{ }^{c}, \overrightarrow{0}(\lambda), \overrightarrow{1}(\lambda)\right\rangle$ is a Boolean algebra, where

$\vec{e} \vee \vec{f}=$ unique $\vec{g} \in A^{n}$ such that $A=\Lambda(\vec{e}, \vec{g}, \vec{f}, \lambda) \wedge R(\vec{e}, \vec{g}, \overrightarrow{1}(\lambda), \lambda)$,

$\vec{e} \wedge \vec{f}=$ unique $\vec{g} \in A^{n}$ such that $A=\Lambda(\vec{e}, \vec{g}, \overrightarrow{0}(\lambda), \lambda) \wedge R(\vec{e}, \vec{g}, \vec{f}, \lambda)$,

$(\vec{e})^{c}=$ unique $\vec{g} \in A^{n}$ such that $A=\Lambda(\vec{e}, \vec{g}, \overrightarrow{1}(\lambda), \lambda) \wedge R(\vec{e}, \vec{g}, \overrightarrow{0}(\lambda), \lambda)$,

$\vec{e} \leq \vec{f}$ iff $A=\Lambda(\vec{f}, \overrightarrow{0}(\lambda), \vec{e}, \lambda)$.

(b) The map

$Z_{\lambda}\left(\Pi\left\{A_{i}: i \in I\right\}\right) \rightarrow \Pi\left\{Z_{\lambda(i)}\left(A_{i}\right): i \in I\right\}$

is an isomorphism.

$$
\vec{e} \quad \rightarrow(\vec{e}(i))_{i \in I}=\left(\left(e_{1}(i), \ldots, e_{n}(i)\right)\right)_{i \in I}
$$

(c) If $\Phi: A \rightarrow B$ is a surjective homomorphism, then the map

$Z_{\lambda}(A) \rightarrow Z_{\Phi(\lambda)}(B)$

is a homomorphism.

$$
\vec{e} \quad \rightarrow \quad \Phi(\vec{e})=\left(\Phi\left(e_{1}\right), \ldots, \Phi\left(e_{n}\right)\right)
$$

(d) If $A \subseteq \Pi\left\{A_{i}: i \in I\right\}$ is a subdirect product, then $Z_{\lambda}(A)$ is a subalgebra of $Z_{\lambda}\left(\Pi\left\{A_{i}: i \in I\right\}\right)$, for every $\lambda \in A$.

Proof. Since $\mathcal{V}$ has the Fraser-Horn property, by $[2,1.4]$, the factor congruences of $A$ form a Boolean sublattice of $\operatorname{Con}(A)$. By Lemma 2, the map $\vec{e} \rightarrow \theta^{A}(\overrightarrow{0}(\lambda), \vec{e})$ is a bijection between $Z_{\lambda}(A)$ and the Boolean algebra of factor congruences of $A$. Thus the partial ordering

$$
\begin{aligned}
\vec{e} \leq \vec{f} \quad \text { iff } & \theta^{A}(\overrightarrow{0}(\lambda), \vec{e}) \subseteq \theta^{A}(\overrightarrow{0}(\lambda), \vec{f}) \\
& \text { iff } \quad A \models \Lambda(\vec{f}, \overrightarrow{0}(\lambda), \vec{e}, \lambda)
\end{aligned}
$$

defines a Boolean algebra structure on $Z_{\lambda}(A)$. At this point we can prove (b) since, by (a) of Lemma 3, the map of (b) is well defined and bijective, and this map and its inverse are order preserving. To prove the remainder of (a), let $\vec{e}, \vec{f} \in Z_{\lambda}(A)$. We can suppose that $A=A_{1} \times A_{2}, \lambda=\left(\lambda_{1}, \lambda_{2}\right)$ and $\vec{e}=\left[\overrightarrow{0}\left(\lambda_{1}\right), \overrightarrow{1}\left(\lambda_{2}\right)\right]$. By (b) $\vec{f}=\left[\vec{f}_{1}, \vec{f}_{2}\right]$ for some $\vec{f}_{k} \in Z_{\lambda_{k}}\left(A_{k}\right), k=1,2$, and

$\vec{e} \vee \vec{f}=\left[\overrightarrow{0}\left(\lambda_{1}\right), \overrightarrow{1}\left(\lambda_{2}\right)\right] \vee\left[\overrightarrow{f_{1}}, \overrightarrow{f_{2}}\right]$

$=\left[\overrightarrow{0}\left(\lambda_{1}\right) \vee \overrightarrow{f_{1}}, \overrightarrow{1}\left(\lambda_{2}\right) \vee \vec{f}_{2}\right]$

$=\left[\overrightarrow{f_{1}}, \overrightarrow{1}\left(\lambda_{2}\right)\right]$

$=$ unique $\vec{g} \in A^{n}$ such that $A=\Lambda(\vec{e}, \vec{g}, \vec{f}, \lambda) \wedge R(\vec{e}, \vec{g}, \overrightarrow{1}(\lambda), \lambda)$.

The other equalities are similar. (c) and (d) follow directly from (a).

We conclude the paper with an example. Let $A=\langle\{0, a, 1\}, \wedge, D, M, 0,1\rangle$, where $\langle\{0, a, 1\}, \wedge, 0,1\rangle$ is the bounded meet semilattice given by the order $0 \leq a \leq 1$, and

$$
\begin{aligned}
& D(x, y)=\left\{\begin{array}{l}
1 \text { if } x=y, \\
0 \text { if } x \neq y,
\end{array}\right. \\
& M(x, y, z)=1, \text { if }\{x, y, z\} \text { has three elements, } \\
& M(x, x, y)=M(x, y, x)=M(y, x, x)=x .
\end{aligned}
$$

Let $\mathcal{V}$ be the variety generated by $A$. Note that $\mathcal{V}$ is a variety with $\overrightarrow{0}$ and $\overrightarrow{1}$ because $\mathcal{V}=0=1 \rightarrow x=y$. Since $M(x, y, z)$ is a majority term, $\mathcal{V}$ is congruence distributive [4].

We will prove that there is no set $\Sigma$ defining the property " $e$ is 0 -central" such that every $\varphi \in \Sigma$ is either universal or existential. Suppose to the contrary that there exists such a set $\Sigma$. Since the language of $\mathcal{V}$ is finite, by Lemma 3 and compactness, we can suppose that $\Sigma$ is finite and hence we can suppose that

$$
\Sigma=\left\{\forall x_{1}, \ldots, x_{m} \psi_{1}\left(x_{1}, \ldots, x_{m}, z\right), \exists x_{1}, \ldots, x_{k} \psi_{2}\left(x_{1}, \ldots, x_{k}, z\right)\right\},
$$


with $\psi_{1}, \psi_{2}$ open formulas. Let $X$ be an infinite set of variables. Since $(0,1) \in$ $F(X) \times F(X)$ is 0 -central, we have that there exist $p_{1}, \ldots, p_{k}, q_{1}, \ldots, q_{k} \in F(X)$ such that

$$
F(X) \times F(X) \models \psi_{2}\left(\left(p_{1}, q_{1}\right), \ldots,\left(p_{k}, q_{k}\right),(0,1)\right) .
$$

Let $x, y$ be elements of $X$ which do not occur in $p_{1}, \ldots, p_{k}, q_{1}, \ldots, q_{k}$. Let $B$ be the subalgebra of $F(X) \times F(X)$ generated by the set

$$
\left\{\left(p_{1}, q_{1}\right), \ldots,\left(p_{k}, q_{k}\right),(0,1)\right\} \cup\{(x, x),(y, y)\} .
$$

Let $\pi_{1}$ and $\pi_{2}$ be the kernels of the canonical projections $B \rightarrow F(X)$. Note that $(0,1)$ is a 0 -central element of $B$. Hence, by modularity, $\theta^{B}((0,0),(0,1))=\pi_{1}$ and $\theta^{B}((1,1),(0,1))=\pi_{2}$, which implies that $(x, y) \in B$. Then there exist a $(k+3)$-ary term $u$ such that

$$
u\left((x, x),(y, y),(0,1),\left(p_{1}, q_{1}\right), \ldots,\left(p_{k}, q_{k}\right)\right)=(x, y),
$$

which implies that

$$
\begin{aligned}
& u\left(x, y, 0, p_{1}, \ldots, p_{k}\right)=x, \\
& u\left(x, y, 1, q_{1}, \ldots, q_{k}\right)=y
\end{aligned}
$$

are identities of $\mathcal{V}$. Note that for every $A \in \mathcal{V}$, the subset $\{0,1\}$ is a subalgebra of $A$. Thus the above identities produce the following ones:

$$
\begin{aligned}
& v(x, y, 0,1)=x, \\
& v(x, y, 1,0)=y,
\end{aligned}
$$

for some term $v$. It is easy to check that if $t$ is a unary term of the language of $\mathcal{V}$, then

$$
A \models t(a)=a \rightarrow t(0)=0,
$$

which for $t=v(1, x, M(x, 0,1), D(M(x, 0,1), 0))$ produces a contradiction.

We observe that in contrast with the above example, in [17] we proved that in a congruence permutable variety with $\overrightarrow{0}$ and $\overrightarrow{1}$ the central elements can be defined in a natural way using $\forall p=q$ axioms. Moreover, the fundamental operations of the Boolean algebra $Z_{\lambda}(A)$ can be expressed by $n$-tuples of terms.

\section{ACKNOWLEDGMENT}

I would like to thank the referee for suggestions which improved the presentation of the paper.

\section{REFERENCES}

[1] J. BERMAN and W. A. BLOK, The Fraser-Horn and apple properties, Trans. Amer. Math. Soc. 302 (1987), 427-465. MR 88h:08010

[2] D. BIGELOW and S. BURRIS, Boolean algebras of factor congruences, Acta Sci. Math., 54 (1990), 11-20. MR 91j:08012

[3] S. BURRIS, Remarks on the Fraser-Horn property, Algebra Universalis, 23 (1986), 19-21. MR 88b:08009

[4] S. BURRIS and H. SANKAPPANAVAR, A Course in Universal Algebra, Springer-Verlag, New York, 1981. MR 83k:08001

[5] C. C. CHANG and H. J. KEISLER, Model Theory, North-Holland, Amsterdam-London, 1973. MR 53:12927

[6] A. DAY, A characterization of modularity for congruence lattices of algebras, Canad. Math. Bull. 12 (1969), 167-173. MR 40:1317

[7] J. DUDA, Fraser-Horn identities can be written in two variables, Algebra Universalis, 26 (1989), 178-180. MR 90d:08009

[8] G. A. FRASER and A. HORN, Congruence relations in direct products, Proc. Amer. Math. 26 (1970), 390-394. MR 42:169

[9] G. GRÄTZER, General Lattice Theory, Birkhäuser Verlag, Basel and Stuttgart, 1978. MR 80c:06001a 
[10] H. P. GUMM, Geometrical Methods in Congruence Modular Algebras, Mem. of the Amer. Math. Soc., 286 (1983). MR 85e:08012

[11] R. McKENZIE, G. McNULTY and W. TAYLOR, Algebras, Lattices, Varieties, Volume 1, The Wadsworth \& Brooks/Cole Math. Series, Monterey, California (1987). MR 88e:08001

[12] J. KOLLAR, Congruences and one element subalgebras, Algebra Universalis, 9 (1979) 266267. MR 80d:08011

[13] R. S. PIERCE, Modules over commutative regular rings, Mem. of the Amer. Math. Soc., 70 (1967). MR 36:151

[14] H. RIEDEL, Existentially closed algebras and Boolean products, Journal of Symbolic Logic 53 (1988), 571-596.

[15] D. VAGGIONE, $\mathcal{V}$ with factorable congruences and $\mathcal{V}=I \Gamma^{a}\left(\mathcal{V}_{D I}\right)$ imply $\mathcal{V}$ is a discriminator variety, Acta Sci. Math. 62 (1996), 359-368. MR 97h:08012

[16] D. VAGGIONE, Varieties in which the Pierce stalks are directly indecomposable, Journal of Algebra 184 (1996), 424-434. MR 97f:08010

[17] D. VAGGIONE, Varieties of shells, Algebra Universalis, 36 (1996) 483-487. MR 97h:08004

Facultad de Matemática, Astronomía y Física (Fa.M.A.F.), Universidad Nacional de Córdoba, Ciudad Universitaria, Córdoba 5000, Argentina

E-mail address: vaggione@mate.uncor.edu 